\title{
Tumor Necrosis Factor Inhibition and Adipose Tissue Distribution - Are Reported Changes Relevant to Cardiometabolic Risk?
}

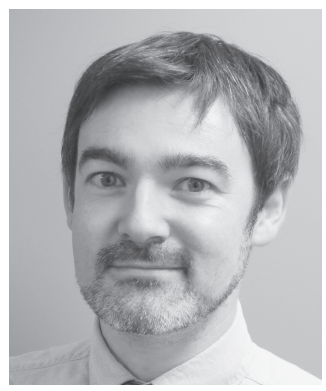

Cardiovascular disease (CVD) is a prominent cause of morbidity and mortality in inflammatory arthritis, including rheumatoid arthritis (RA), psoriatic arthritis (PsA), and ankylosing spondylitis (AS), while traditional CVD risk factors, such as hypertension and dyslipidemia, are more prevalent in these patients ${ }^{1,2}$. In the general population, obesity is recognized as a risk factor for hypertension, dyslipidemia, insulin resistance, and the metabolic syndrome (MetS), the so-called "cardiometabolic" risk factors; however, obesity has not been shown to improve risk prediction of CVD compared with those variables currently included in risk prediction models ${ }^{3}$.

The distribution of body fat may have greater influence on CVD risk, particularly that located in the visceral compartments (visceral adipose tissue, or VAT). In the general population, VAT has been associated with CVD events $^{4}$, coronary artery calcification ${ }^{5}$, and aortic stiffness ${ }^{6}$. In RA, VAT area higher than the 75 th percentile was associated with a significantly higher probability of hypertension, raised fasting glucose, and MetS ${ }^{7}$. It may also predispose to insulin resistance and expresses a proinflammatory profile of $\mathrm{C}$-reactive protein, tumor necrosis factor$\alpha$ (TNF- $\alpha$ ), and interleukin 6 expression ${ }^{8}$. Fox and colleagues advise that the contribution of subcutaneous adipose tissue (SAT), which accounts for $80 \%$ of the body's fat, may also play a relevant role in CVD risk. A large crosssectional study examining the association of VAT and SAT with metabolic risk factors in 3001 Third Generation and Offspring participants of the Framingham Heart Study found that waist circumference, triglycerides, high-density lipoprotein (HDL), glucose, and blood pressure all had a significant correlation with both VAT and SAT, although the correlations were stronger with $\mathrm{VAT}^{9}$.

Surrogate measures of adiposity such as body mass index (BMI), waist circumference (WC), and waist-to-hip ratio (WHR) do not accurately reflect body composition. In an RA population where VAT area was significantly greater than controls, BMI, WC, and WHR were similar between the 2 groups ${ }^{7}$, suggesting that anthropometric measures are not an equivalent to specific fatty tissue quantification.
Computed tomography and magnetic resonance imaging generate the most accurate data for VAT quantification, but their cost and accessibility limit their use. For those reasons, dual-energy $\mathrm{x}$-ray absorptiometry (DEXA) is a useful alternative in assessing body composition ${ }^{10}$.

In this issue of The Journal, Hmamouchi and coworkers report their findings from an uncontrolled prospective study conducted over 2 years at a single tertiary center in Paris, France ${ }^{11}$. The authors examined the effect of TNF inhibitor (TNFi) therapy on BMI, waist circumference, fat and lean mass, and VAT and SAT in 85 patients with spondyloarthritis (SpA; 79 with AS and 6 with peripheral SpA). DEXA was used to determine fat mass, lean mass, VAT area, and SAT area. There were significant increases from baseline in BMI, waist circumference, and VAT and SAT after 2 years of TNFi therapy, with no apparent effect of age or inflammatory markers on these increases. The authors also report findings from a group of 18 patients with VAT and SAT measurements 8 months before initiating TNFi. Interestingly, there was no significant change in SAT in the pre-TNFi period up to TNFi initiation and after 6 and 12 months of treatment. Similarly with VAT, no significant difference was found in the VAT area measured in the period before TNFi and in the first 6 months of treatment.

The authors' findings raise important methodological considerations in studies examining anthropometric changes in both the general population and in those relating to rheumatic disease. Large epidemiological studies have shown that after the age of 30, the weight of both sexes increases over time and starts to decline in the sixth decade ${ }^{12,13}$. Fox, et al demonstrated that VAT volumes were positively correlated with age in both sexes ${ }^{9}$. Although Hmamouchi and coworkers found no relationship between it and increasing adipose area and BMI, the effect of age is an important consideration.

In the larger cross-sectional studies examining fat and lean mass in AS, no differences have been observed between patients and controls ${ }^{14,15}$. A comparison of body composition using DEXA between 30 patients with RA, 31 patients with AS, and 51 healthy controls found that BMI

See Abdominal adiposity in SpA treated with anti-TNF, page 1112 
was similar in all 3 groups. There was a trend for higher lean mass and lower fat mass in the AS group, while in RA, fat mass was increased with greater VAT area in female patients compared with controls ${ }^{16}$. Because there was no accumulation of visceral fat in the AS group in Toussirot's study ${ }^{16}$, Hmamouchi's findings of VAT escalation in those receiving TNFi therapy suggest that TNFi could induce changes in body composition. A controlled, prospective study would assist in clarifying this.

Reversal of the catabolic effects of TNF- $\alpha$, such as weight loss, anorexia, proteolysis, and downregulation of anabolic hormones through targeted blockade of the cytokine, could result in weight gain. Alternatively, reduced joint pain and stiffness might allow for an increase in physical activity, with associated weight loss. Weight gain and change in adipose tissue distribution have been reported in those prescribed TNFi in rheumatic disease. In an AS population from the same group as Hmamouchi, Briot, et al reported an increase of $3.4 \%$ in weight over 12 months while taking TNFi, a $12.1 \%$ increase in fat mass and a $1.9 \%$ increase in lean mass, while bone mineral density also increased ${ }^{17}$. Weight gain occurred primarily within the first 6 months of initiating TNFi treatment. In psoriatic disease, a number of studies have reported weight increase with TNFi use ${ }^{18,19,20}$; however, those studies have collectively failed to account for the natural tendency for weight gain and alteration of adipose tissue distribution with age. Our group, reporting from a PsA longitudinal cohort study, accommodated this natural change by examining weight gain in the period prior to TNFi prescription (over an average of $4.4 \mathrm{yrs}$ ) compared to after TNFi initiation (over an average of $5 \mathrm{yrs}$ ), and found no significant difference ${ }^{21}$.

Whether TNFi induces an adverse cardiometabolic risk profile through excess VAT deposition remains unanswered. In a population with RA of recent onset, Engvall and colleagues found a significant increase in fat mass after commencement of infliximab (3.8 kg) compared to a combination of sulfasalazine and hydroxychloroquine $(0.4 \mathrm{~kg})$ over 24 months, with a similar reduction in disease activity. Encouragingly, there was no change in the lipidemic profile despite the increase in fat mass ${ }^{22}$. Also in RA, TNFi therapy over 12 weeks was shown to improve insulin sensitivity, increase HDL and reduce triglyceride levels ${ }^{23}$. In PsA and AS, surrogates of atherosclerosis and endothelial dysfunction have shown improvement following treatment with $\mathrm{TNFi}^{1,2}$.

Do TNF inhibitors cause an increase in proinflammatory, proatherosclerotic VAT in SpA? This has not yet been determined; however, the findings reported by Hmamouchi and colleagues warrant further study. Examination of cohorts from longitudinal studies would facilitate assessment of fat mass and adipose distribution change and the effect of age/time on preintervention and postintervention with TNFi. The effect of changes in VAT and SAT measurements on CVD morbidity, mortality, and related surrogate markers needs to be elucidated, as does the potential for reduced drug efficacy, given that obese patients with PsA are 5 times less likely to achieve minimal disease activity with $\mathrm{TNFi}^{24}$.

Until we have answers, recommendations for cardiovascular risk management in rheumatic disease provided by EULAR $^{25}$ and other regional rheumatology authorities should be adhered to.

BARRY J. SHEANE, MB, BCh, BAO,

Centre for Prognosis Studies in the Rheumatic Diseases, Toronto Western Hospital,

399 Bathurst Street,

Toronto, Ontario, Canada M5T 2S8

Address correspondence to Dr. Sheane; E-mail: sheaneb@yahoo.com

\section{REFERENCES}

1. Jamnitski A, Symmons D, Peters MJ, Sattar N, McInnes I, Nurmohamed MT. Cardiovascular comorbidities in patients with psoriatic arthritis: a systematic review. Ann Rheum Dis 2013;72:211-6.

2. Nurmohamed MT, van der Horst-Bruinsma I, Maksymowych WP. Cardiovascular and cerebrovascular diseases in ankylosing spondylitis: current insights. Curr Rheumatol Rep 2012;14:415-21.

3. Cameron AJ, Magliano DJ, Soderberg S. A systematic review of the impact of including both waist and hip circumference in risk models for cardiovascular diseases, diabetes and mortality. Obes Rev 2013;14:86-94.

4. Mahabadi AA, Massaro JM, Rosito GA, Levy D, Murabito JM, Wolf PA, et al. Association of pericardial fat, intrathoracic fat, and visceral abdominal fat with cardiovascular disease burden: the Framingham Heart Study. Eur Heart J 2009;30:850-6.

5. Rosito GA, Massaro JM, Hoffmann U, Ruberg FL, Mahabadi AA, Vasan RS, et al. Pericardial fat, visceral abdominal fat, cardiovascular disease risk factors, and vascular calcification in a community-based sample: the Framingham Heart Study. Circulation 2008;117:605-13.

6. Sutton-Tyrrell K, Newman A, Simonsick EM, Havlik R, Pahor M, Lakatta $\mathrm{E}$, et al. Aortic stiffness is associated with visceral adiposity in older adults enrolled in the study of health, aging, and body composition. Hypertension 2001;38:429-33.

7. Giles JT, Allison M, Blumenthal RS, Post W, Gelber AC, Petri M, et al. Abdominal adiposity in rheumatoid arthritis: association with cardiometabolic risk factors and disease characteristics. Arthritis Rheum 2010;62:3173-82.

8. Ibrahim MM. Subcutaneous and visceral adipose tissue: structural and functional differences. Obes Rev 2010;11:11-8.

9. Fox CS, Massaro JM, Hoffmann U, Pou KM, Maurovich-Horvat P, Liu CY, et al. Abdominal visceral and subcutaneous adipose tissue compartments: association with metabolic risk factors in the Framingham Heart Study. Circulation 2007;11639-48.

10. Shuster A, Patlas M, Pinthus JH, Mourtzakis M. The clinical importance of visceral adiposity: a critical review of methods for visceral adipose tissue analysis. Br J Radiol 2012;85:1-10

11. Hmamouchi I, Roux C, Paternotte S, Kolta S, Dougados M, Briot $\mathrm{K}$. Early increase in abdominal adiposity in patients with spondyloarthritis receiving anti-tumor necrosis factor alpha treatment. J Rheumatol 2014;41:1112-7.

12. Williamson DF. Descriptive epidemiology of body weight and weight change in U.S. adults. Ann Intern Med 1993;119:646-9.

13. Droyvold WB, Nilsen TI, Kruger O, Holmen TL, Krokstad S, Midthjell K, et al. Change in height, weight and body mass index: Longitudinal data from the HUNT Study in Norway. Int J Obes 
2006;30:935-9.

14. Toussirot E, Michel F, Wendling D. Bone density, ultrasound measurements and body composition in early ankylosing spondylitis. Rheumatology 2001;40:882-8.

15. Plasqui G, Boonen A, Geusens P, Kroot EJ, Starmans M, van der Linden S. Physical activity and body composition in patients with ankylosing spondylitis. Arthritis Care Res 2012;64:101-7.

16. Toussirot E, Grandclement E, Gaugler B, Michel F, Wendling D, Saas $\mathrm{P}$, et al. Serum adipokines and adipose tissue distribution in rheumatoid arthritis and ankylosing spondylitis. A comparative study. Front Immunol 2013;4:453.

17. Briot K, Gossec L, Kolta S, Dougados M, Roux C. Prospective assessment of body weight, body composition, and bone density changes in patients with spondyloarthropathy receiving anti-tumor necrosis factor-alpha treatment. J Rheumatol 2008;35:855-61.

18. Gisondi P, Cotena C, Tessari G, Girolomoni G. Anti-tumour necrosis factor-alpha therapy increases body weight in patients with chronic plaque psoriasis: a retrospective cohort study. J Eur Acad Dermatol Venereol 2008;22:341-4.

19. Saraceno R, Schipani C, Mazzotta A, Esposito M, Di Renzo L, De Lorenzo A, et al. Effect of anti-tumor necrosis factor-alpha therapies on body mass index in patients with psoriasis. Pharmacological Res 2008;57:290-5.

20. Renzo LD, Saraceno R, Schipani C, Rizzo M, Bianchi A, Noce A, et al. Prospective assessment of body weight and body composition changes in patients with psoriasis receiving anti-TNF-alpha treatment. Dermatol Ther 2011;24:446-51.

21. Sheane BJ, Thavaneswaran A, Gladman DD, Chandran V. Changes in weight associated with tumour necrosis factor inhibition in psoriatic arthritis [abstract]. Arthritis Rheum 2013;65 Suppl 10:S144.

22. Engvall IL, Tengstrand B, Brismar K, Hafstrom I. Infliximab therapy increases body fat mass in early rheumatoid arthritis independently of changes in disease activity and levels of leptin and adiponectin: a randomised study over 21 months. Arthritis Res Ther 2010;12:R197.

23. Stagakis I, Bertsias G, Karvounaris S, Kavousanaki M, Virla D, Raptopoulou A, et al. Anti-tumor necrosis factor therapy improves insulin resistance, beta cell function and insulin signaling in active rheumatoid arthritis patients with high insulin resistance. Arthritis Res Ther 2012;14:R141.

24. di Minno MN, Peluso R, Iervolino S, Lupoli R, Russolillo A, Scarpa $R$, et al. Obesity and the prediction of minimal disease activity: a prospective study in psoriatic arthritis. Arthritis Care Res 2013;65:141-7.

25. Peters MJ, Symmons DP, McCarey D, Dijkmans BA, Nicola P, Kvien TK, et al. EULAR evidence-based recommendations for cardiovascular risk management in patients with rheumatoid arthritis and other forms of inflammatory arthritis. Ann Rheum Dis 2010;69:325-31

J Rheumatol 2014;41:1035-7; doi:10.3899/jrheum.140439 